Joël Belmin

\section{Alain Tedgui}

\title{
Le vieillissement de la paroi artérielle
}

Au cours du vieillissement, se produit un remodelage progressif des artères, caractérisé par une augmentation du calibre des gros troncs et un remaniement de la paroi. Les fibres élastiques de la paroi sont altérées et le contenu en collagène augmente. Le collagène, qui subit une glycosylation non enzymatique, devient plus rigide et plus résistant à la protéolyse. Les cellules musculaires lisses acquièrent un phénotype " jeune ", c'està-dire prolifératif et sécrétoire, qui contribue au remaniement fibreux et à l'hypertrophie de la paroi. L'espace sous-endothélial s'épaissit considérablement. Cette couche est constituée de collagène, de protéoglycanes, de cellules musculaires lisses et de cellules mononucléées, normalement absentes dans cette zone. Certaines fonctions endothéliales sont altérées, comme la relaxation dépendante de l'endothélium, la production de prostacycline, et la fonction de barrière. Ce remodelage de la paroi pourrait être dû à des facteurs mécaniques, à l'action de cytokines, de facteurs de croissance, et/ou de protéases. C'est sur le substratum d'une paroi artérielle ainsi modifiée que se développent certaines maladies vasculaires particulièrement fréquentes chez les sujets âgés, telles que l'hypertension et l'athérosclérose.

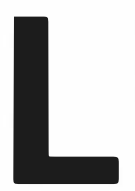

e vieillissement s'accompagne de modifications importantes du système artériel qui constituent le trait le plus marquant du vieillissement de l'appareil cardiovasculaire. $\mathrm{Si}$ les modifications morphologiques des artères qui surviennent au cours $\mathrm{du}$ vieillissement sont connues de longue date, d'autres aspects du vieillissement de la paroi ont été découverts plus récemment, à la suite des progrès réalisés dans le domaine de la biologie de la paroi artérielle.

La recherche sur le vieillissement de \begin{abstract}
la paroi artérielle se heurte à certai-
\end{abstract}
nes difficultés inhérentes à la recherche en gérontologie, liées notamment, d'une part, aux modèles expérimentaux et, d'autre part, à la distinction entre maladies liées à l'âge et vieillissement physiologique. En particulier, l'athérosclérose, dont le lien statistique avec l'âge est frappant [1-3], a été souvent confondue avec le vieillissement artériel. Si ces deux processus peuvent être associés ou interagir l'un sur l'autre, il est clair qu'ils sont de nature différente. L'athérosclérose est une maladie localisée, qui s'accompagne d'une diminution du calibre artériel, très différente du
U. 141, hôpital Lariboisière, 41, boulevard de la Chapelle, 75010 Paris, France.
$J$. Belmin : praticien hospitalier, chef du service de gérontologie médicale, hôpital René-MuretBigottini, avenue du Docteur-Schaeffner, 93270 Sevran, France.

A. Tedgui : directeur de recherche Inserm, de la Chapelle, 75010 Paris, France. 
Figure 1. Évolution en fonction de I'âge de la morphologie de l'aorte humaine, étudiée à partir d'autopsies effectuées en Australie et aux États-Unis. Les aortes étaient fixées sous une pression de $100 \mathrm{mmHg}$ et la circonférence (A), I'épaisseur de l'intima (B) et de la média (C) étaient mesurées par histomorphométrie. Thor desc : thoracique descendante; abd : abdominale. (D'après Virmani et al. [5].)

vieillissement artériel qui est un processus physiologique, diffus, et qui entraîne un élargissement de la lumière artérielle des gros vaisseaux. En revanche, l'artériosclérose qui est un processus diffus, et qui ne semble pas pouvoir à elle seule entraîner de manifestations pathologiques, peut être assimilée à une forme extrême de vieillissement artériel.

\section{Morphologie des artères}

$\mathrm{Au}$ cours du vieillissement, l'aspect morphologique des artères change. Les diamètres artériels interne et externe des grosses artères de type élastique augmentent de façon importante (figures 1 et 2), ainsi que la longueur des artères, d'où l'apparition de tortuosité artérielle [4, 5]. En revanche, dans les petites artères de type musculaire, le diamètre externe est peu modifié au cours du vieillissement, alors que le diamètre interne diminue, essentiellement du fait de l'épaississement intimal.

De façon générale, la paroi artérielle s'épaissit au cours du vieillissement, l'hypertrophie atteignant surtout l'intima et, à un moindre degré, la média (figure 1) [6]. L'épaississement de l'intima lié à l'âge est considérable (figure 1) et l'épaisseur de l'intima, mesurée par histomorphométrie, peut être multipliée par un facteur 4 à 10 par rapport à celle d'artères de sujets plus jeunes [7, 8]. En fait, la plupart des composants cellulaires et extracellulaires de la paroi artérielle semblent modifiés par le vieillissement.

\section{Modifications cellulaires}

\section{Les cellules endothéliales}

Le vieillissement ne semble pas modifier de façon importante l'aspect mor$\mathrm{m} / \mathrm{s} n^{\circ} 10 \mathrm{vol} .9$, octobre 93
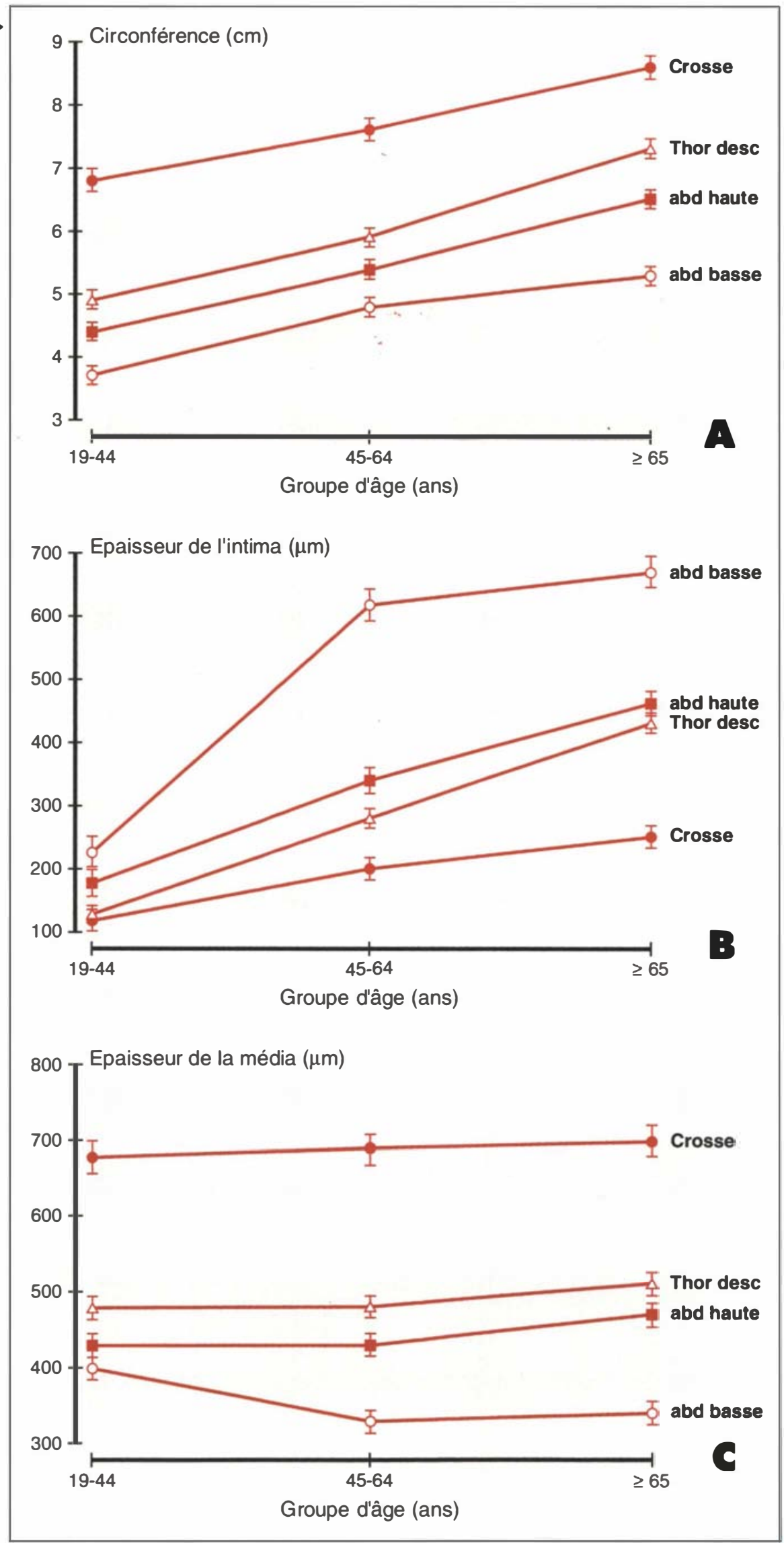


\section{RÉFÉRENCES}

1. Bates SR, Gancloff EC. Atherosclerosis and aging. New York: Springer-Verlag, 1987.

2. Weber G, Bianciardi G, Bussani R, et al. Atherosclerosis and aging : a morphometric study on arterial lesions of elderly and very elderly necropsy subjects. Arch Pathol Lab Med 1988; 112 : 1066-70.

3. Kannel WP, Gordon T. Evaluation of cardiovascular risk in the elderly : the Fra mingham study. Bull N Y Acad Med 1978 ; $54: 573-91$

4. Bouissou $\mathbf{H}$, Pieraggi MT, Julian $\mathbf{M}$ Variations morphologiques avec l'âge. In : Camillieri JP, Berry C, Fiessinger JN, Bariety J, eds. Maladies de la paroi artérielle. Paris : Flammarion, 1987 : 60-6.

5. Virmani R, Avolio AP, Mergner WJ, et al. Effect of aging on aortic morphology in populations with high and low prevalence of hypertension and atherosclerosis. $A m \mathrm{~J}$ Pathol $1991 ; 139$ : 1119-29.

6. Wolinsky H. Long-term effects of hypertension on the rat aortic wall and their relation to concurrent aging changes. Morphological and chemical studies. Circ Res 1972 ; 30 : 301-9.

7. Gerrity RG, Cliff WF. The aortic tunical intimal in young and aging rats. Exp Mol Pathol 1972 ; 16 : 382-402.

8. Guyton JR, Lindsay KL, Dao DT Comparison of aortic intima and inner media in young adult versus aging rats. $\mathrm{Am}$ $J$ Pathol 1983; 111: 234-46.

9. Repin VS, Dolgov VV, Zaikina OE, et al. Heterogeneity of endothelium in human aorta. A quantitative analysis by scanning electron microscopy. Atherosclerosis 1984 ; 50 : 35-52.

10. Tokunaga O, Fan J, Watanabe $T$. Atherosclerosis and age-related multinucleated variant endothelial cells in primary culture from human aorta. Am J Pathol 1989 ; 135 : 967-76.

11. Schwartz MS, Benditt EP. Aortic endothelial cell replication. I. Effects of age and hypertension in the rat. Circ Res $1977 ; 41$ : 248-55.

12. Hongo $\mathrm{K}$, Nakagomi $\mathrm{T}$, Kassell NF, et al. Effects of aging and hypertension on endothelium-dependent vascular relaxation in rat carotid artery. Stroke 1988; 19 892-7.

13. Koga $T$, Takata $Y$, Kobayashi K, Takishita S, Yamashita Y, Fujishima M. Aging supresses endothelium-dependent relaxation and generates contraction mediated by the muscarinic receptors in vascular smooth muscle of normotensive WistarKyoto and spontaneously hypertensive rats. $J$ Hypertens $1988 ; 6$ : S243-5.

14. Soltis EE. Effect of age on blood pres- sure and membrane-dependent vascular responses in the rat. Circ Res $1987 ; 10$ : 889-97.

15. Yasue H, Matsuyama K, Matsuyama K, Okumura K, Morikami Y, Ogawa H. Responses of angiographically normal human coronary arteries to intracoronary injection of acetylcholine by age and segment. Circulation 1990 ; 81 : 482-90.

16. Ishihata $\mathrm{A}, \mathrm{Katano} \mathrm{Y}$, Morinobu $\mathrm{S}$, Endoh M. Influence of aging on the contractile response to endothelin of rat thoracic aorta. Eur J Pharmacol 1991; 200 : 199-201.

17. Tokunaga O, Yamada T, Fan J, Watanabe $\mathrm{T}$. Age-related decline in prostacyclin synthesis by human aortic endothelial cells. Am J Pathol 1991; 138 : 941-9.

18. Esposito C, Gerlag H, Brett J, Stern D, Vlassara H. Endothelial receptor-mediated binding of glucose-modified albumin is associated with increased monolayer permeability and modulation of cell surface coagulant propertics. $J$ Exp Med 1989; 170 : 1387-407.

19. Belmin J, Corman B, Merval R, Tedgui A. Age-related changes in albumin endothelial permeability and distribution volume in the rat aorta. Am J Physiol 1993 ; 264: 679-85.

20. Cliff WJ. The aortic tunica media in aging rats. Exp Mol Pathol 1970; 13 : 172-89.

21. McCaffrey TA, Nicholson AC, Szabo PE, Weksler ME, Weksler BB. Aging and arteriosclerosis. The increased proliferation of arterial smooth muscle cells isolated from old rats is associated with increased PlateletDerived Growth Factor-like activity. I Exp Med 1988 ; 167 : 163-74.

22. Hariri RJ, Alonso DR, Hajjar DP, Coletti D, Weksler ME. Aging and arteriosclerosis. Development of myointimal hyperplasia after endothelial injury. $J$ Exp Med $1986 ; 164$ : 1171-8.

23. Hariri RJ, Hajjar DP, Coletti D, Alonso DR, Weksler ME, Rabellino E. Aging and arteriosclerosis. Cell cycle kinetics of young and old arterial. Smooth muscle cells. Am $J$ Pathol 1988 ; 131 : 132-6.

24. Schwartz S. Growth factors and the vessel wall. Prog Hemost Thromb 1982; 6 : 85-112.

25. Hayflick L, Moorhead PS. The serial cultivation of human diploid cell strains. Exp Cell Res 1961; 25 : 585-621.

26. Courtois Y. Vieillissement cellulaire et moléculaire. In : Bourlière F, ed. Gérontologie biologique et moléculaire. Paris : Flammarion, $1982:$ 5-25. phologique des cellules endothéliales. Toutefois, certains auteurs ont rapporté chez l'homme âgé une proportion importante de cellules endothéliales géantes et multinucléées $[9,10]$. La quantité de réticulum endoplasmique rugueux des cellules endothéliales, qui reflète l'activité de leur synthèse protéique, augmente chez les rats âgés [8]. L'augmentation de cette activité synthétique pourrait contribuer à l'apparition de matériel fibreux dans l'intima, et notamment d'élastine microfibrillaire et de protéoglycanes. En outre, la synthèse d'ADN dans l'endothélium augmente avec l'âge, correspondant à une augmentation du taux de renouvellement des cellules endothéliales, plutôt qu'à une prolifération accrue [11].

Les fonctions endothéliales semblent, elles aussi, être modifiées au cours du vieillissement. La capacité de l'endothélium à sécréter des substances vasodilatatrices est altérée. La relaxation dépendante de l'endothélium, induite par l'EDRF (endothelium dependent relaxing factor), est nettement diminuée chez les animaux [12-14] et l'homme [15] âgés (figure 3). Cette diminution n'est pas expliquée par des anomalies des récepteurs endothéliaux de l'acétylcholine [14]. En revanche, la sécrétion d'EDRF par l'endothélium pourrait être diminuée avec l'avancée en âge, et/ou l'EDRF sécrété aurait plus de difficulté à atteindre les cellules musculaires lisses pour y exercer son effet relaxant, du fait de l'épaississement intimal lié à l'âge et de sa courte demivie [12-14]. De plus, l'action modulatrice de l'endothélium, qui atténue l'effet vasoconstricteur de l'endothéline, est aussi diminuée chez le rat âgé [16]. La libération de prostaglandine PGI2 par les cellules endothéliales en culture provenant d'hommes âgés est diminuée [17]. Enfin, le vieillissement s'accompagne d'une augmentation de la perméabilité endothéliale vis-à-vis des macromolécules plasmatiques, aussi bien sur des modèles de couches unicellulaires de cellules endothéliales en culture [18], que chez le rat in vivo (figure 4) [19]. Ainsi, au cours du vieillissement, la fonction de barrière de l'endothélium est altérée. De ce fait, il est probable que les macromolécules plasmatiques, ainsi que certaines cellules cir- 


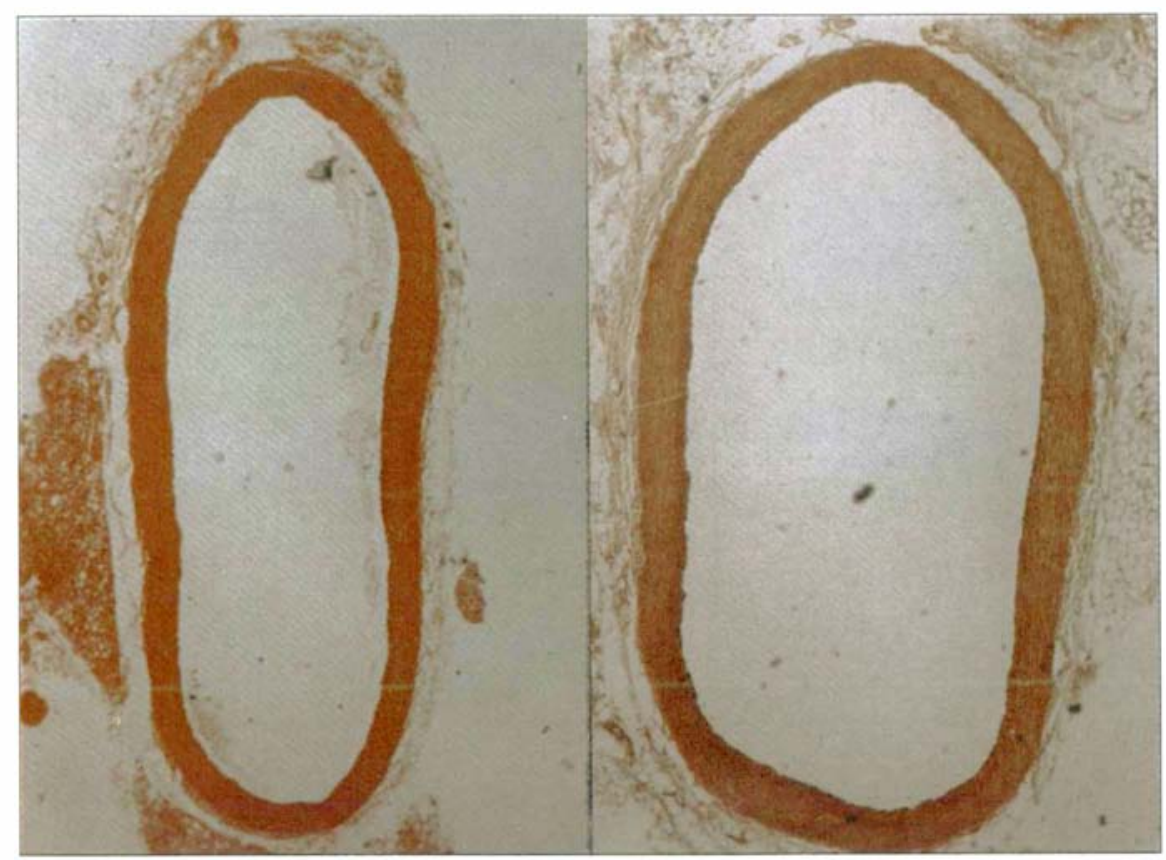

Figure 2. Aorte thoracique de rats de 10 mois (A) et de 30 mois (B). Les circonférences interne et externe sont augmentées chez le rat âgé, ainsi que la surface de la lumière. L'épaisseur totale de l'aorte est modérément augmentée chez le rat âgé, et le volume total de tissu aortique est augmenté.

culantes, ont un accès plus facile à l'espace sous-endothélial et à la média chez les animaux âgés.

\section{Les cellules musculaires lisses}

La densité des cellules musculaires lisses diminue au cours de la croissance, puis se stabilise au cours du vieillissement, tandis que le processus d'hypertrophie pariétale paraît plus continu [20]. On constate donc une augmentation de la masse cellulaire au cours du vieillissement, associée à une hypertrophie des cellules musculaires lisses elles-mêmes. Les études menées sur des cultures cellulaires ont montré que les cellules musculaires lisses de rats âgés croissent plus rapidement que celles des jeunes rats [21]. Cette activité prolifératrice accrue est associée à une production accrue de PDGF (platelet derived growth factor) et à une diminution de la sécrétion de facteurs inhibiteurs tels que les héparane-sulfates. Les lysats de cellules musculaires lisses âgées sont capables de stimuler la prolifération de cellules cibles en culture, beaucoup plus facilement que les lysats de cellules musculaires lisses $m / s n^{\circ} 10$ vol. 9 , octobre 93 de cellules jeunes se trouve dans la phase $G_{0} / G_{1}$, tandis qu'une proportion plus grande se situe dans la phase S [23, 24]. Cette différence dans la distribution des cellules dans le cycle pourrait refléter un état phénotypique différent des cellules musculaires lisses âgées. On sait en effet que lorsque les cellules musculaires lisses évoluent d'un phénotype " contractile " (état des cellules musculaires lisses quiescentes de la paroi adulte normale) à un phénotype "synthétique " (cellules musculaires lisses proliférantes myo-intimales), elles répondent plus facilement à une stimulation mitogène. Le vieillissement artériel pourrait donc s'accompagner d'une dédifférenciation des cellules musculaires lisses de la média, celles-ci acquérant à nouveau le phénotype synthétique à potentiel prolifératif amplifié qui caractérise les cellules musculaires lisses de la paroi artérielle du fæetus ou du nouveau-né.

La réponse des cellules musculaires lisses au chlorure de potassium [16] et à des agents vasoconstricteurs comme l'adrénaline ou la 5-hydroxytryptamine n'est pas modifiée par l'âge $[12,13,16]$. Toutefois, la vasoconstriction induite par l'endothéline est diminuée chez les rats âgés [16]. La réponse des cellules musculaires lisses à des vasodilatateurs comme le nitroprussiate [13], ou la papavérine, n'est pas modifiée par l'âge [12].

\section{Autres cellules}

Le vieillissement in vivo des fibroblastes de la paroi artérielle est mal connu. Les connaissances relatives au vieillissement des fibroblastes en culture ne sont sans doute pas extrapolables aux fibroblastes de la paroi in vivo. En effet, en culture, ces cellules se divisent jusqu'à épuisement de leur potentiel de division (phénomène de Hayflick) ([25], voir revue de Courtois [26]), alors que les phénomènes de prolifération des fibroblastes sont rarement observés dans la paroi. Cette modulation phénotypique des fibroblastes semble liée aux interactions cellules-matrice, car les fibroblastes en culture placés dans un gel de collagène tridimensionnel adoptent un comportement non prolifératif.

Des cellules mononucléées sont observées dans l'intima des artères âgées, 


\section{RÉFÉRENCES}

27. Haudenschild CC, Prescott MF, Chobanian AV. Aortic endothelial and subendothelial cells in experimental hypertension and aging. Hypertension 1981; 3 : I148-53.

28. Brownlee $M$, Vlassara $\mathrm{H}$, Cerami A. Nonenzymatic glycosylation and the pathogenesis of diabetic complications. Ann Intern Med 1984； 101 : 527-37.

29. Vlassara $H$, Brownlee $M$, Manogue KR, Dinarello CA, Pasaglan A. Cachectin/TNF and IL-1 induced by glucosemodified proteins : role in normal tissue remodeling. Science $1988 ; 240: 1546-8$.

30. Robert L, Jacob MP, Frances C, Godeau $G$, Hornebeck $W$. Interaction between elastin and elastases and its role in aging of arterial wall, skin, and other connective tissues. A review. Mech Aging Dev $1984 ; 28: 155-66$.

31. Fischer GM. Effects of spontaneous hypertension and age on arterial connective tissue in the rat. Exp Gerontol 1976; 11: 209-15.

32. Rucker RB, Tinker D. Structure and metabolism of arterial elastin. Int Rev Exp Pathol $1977 ; 17: 1-47$

33. McMahon MP, Faris B, Wolfe BL, et al. Aging effects on the elastin composition in the extracellular matrix of cultured rat aortic smooth muscle cells. In Vitro 1985 ; 21: 674-80.

34. Schinder SL, Kohn RR. Glycosylation of human collagen in aging and diabetes mellitus. J Clin Invest 1980 ; 66 : 1179-81.

35. Sell DR, Monnier VM. Structure elucidation of a senescence cross-link from human extracellular matrix. J Biol Chem $1989 ; 264: 21597-602$.

36. Richardson M, Hatton MWC, Moore $\mathrm{S}$. Proteoglycan distribution in the intima and media of the aortas of young and aging rabbits : an ultrastructural study. Atherosclerosis $1988 ; 71: 243-56$

37. Jackson RL, Busch $\mathrm{SJ}$, Cardin AD. Glycosaminoglycans : molecular properties, interactions and role in physiological processes. Physiol Rev 1991; 71: 481-526.

38. Beisielgel U, Niendorf A, Wolf $K$, Reblin T, Rath M. Lipoprotein (a) in the arterial wall. Eur Heart J 1990 ; 11 : 174-83.

39. Michel RP. Hypertension artćrielle pulmonaire. In : Camillieri JP, Berry C, Fiessinger JN, Bariety J, eds. Maladies de la paroi artérielle. Paris : Flammarion, 1987 ; 547-75.

40. Feldman SA, Glagov S. Transmedial collagen and elastin gradients in human aortas : reversal with age. Atherosclerosis 1971 . $13: 385-94$.

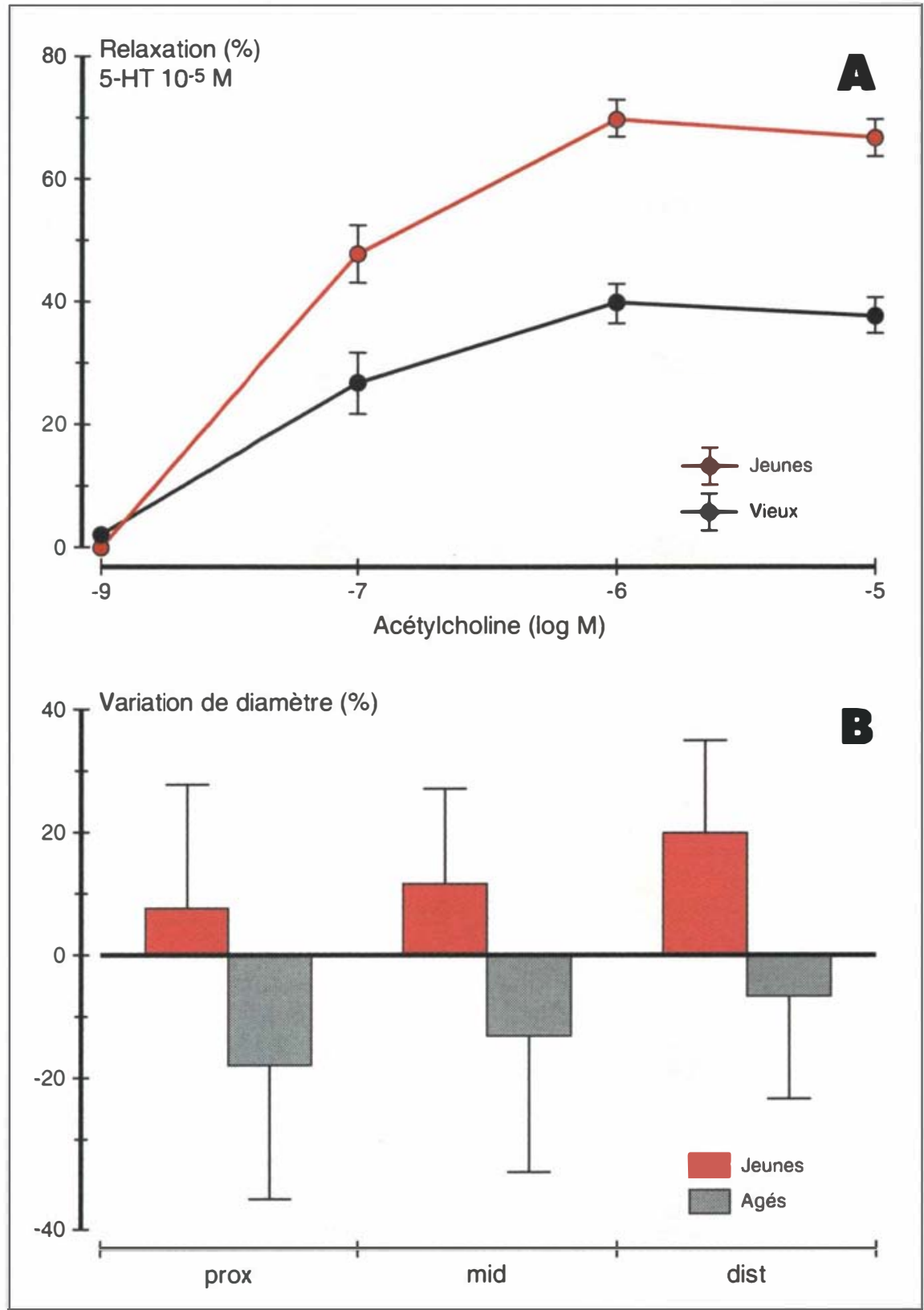

Figure 3. Relaxation dépendante de l'endothélium induite par l'acétylcholine d'anneaux aortiques provenant de rats jeunes et âgés (A), et d'artères coronaires humaines jeunes et âgées (B). La réponse vasodilatatrice à l'acétylcholine est diminuée chez le rat âgé, et se trouve abolie dans les artères coronaires humaines, laissant place à une vasoconstriction. (D'après Hongo et al. [12] (A), et Yasue et al. [15] (B).) 


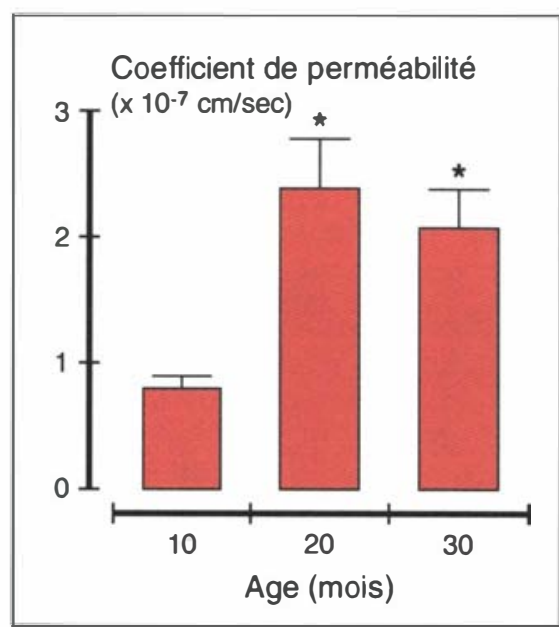

Figure 4. Évolution en fonction de l'âge du coefficient apparent de perméabilité endothéliale à l'albumine étudié in vivo dans l'aorte de rat, en mesurant les concentrations transmurales d'albumine-l'131, 5 min après I'injection intraveineuse de ce traceur. ( $D$ 'après Belmin et al. [19].) sans que cela corresponde à un phénomène pathologique lié à l'athérosclérose [7, 8, 27]. Ces cellules sont absentes ou très rares dans la paroi artérielle d'adultes jeunes. Le rôle de ces lymphocytes et macrophages dans l'intima est mal connu. Ils pourraient intervenir dans des processus de phagocytose, ou encore moduler l'activité des cellules musculaires lisses en libérant des cytokines ou d'autres facteurs contrôlant l'activité cellulaire. En effet, les macrophages de la paroi disposent de récepteurs leur permettant de reconnaître les protéines qui ont subi une glycation (encore appelée glycosylation) non enzymatique, protéines qui s'accumulent au cours du vieillissement $[18,28]$; la liaison à ce récepteur entraîne une sécrétion de facteurs immuno-modulateurs tels que le TNF (tumor necrosis factor) et l'IL1 (interleukine 1) qui peuvent, à leur tour, induire des processus de dégradation (en induisant la sécrétion

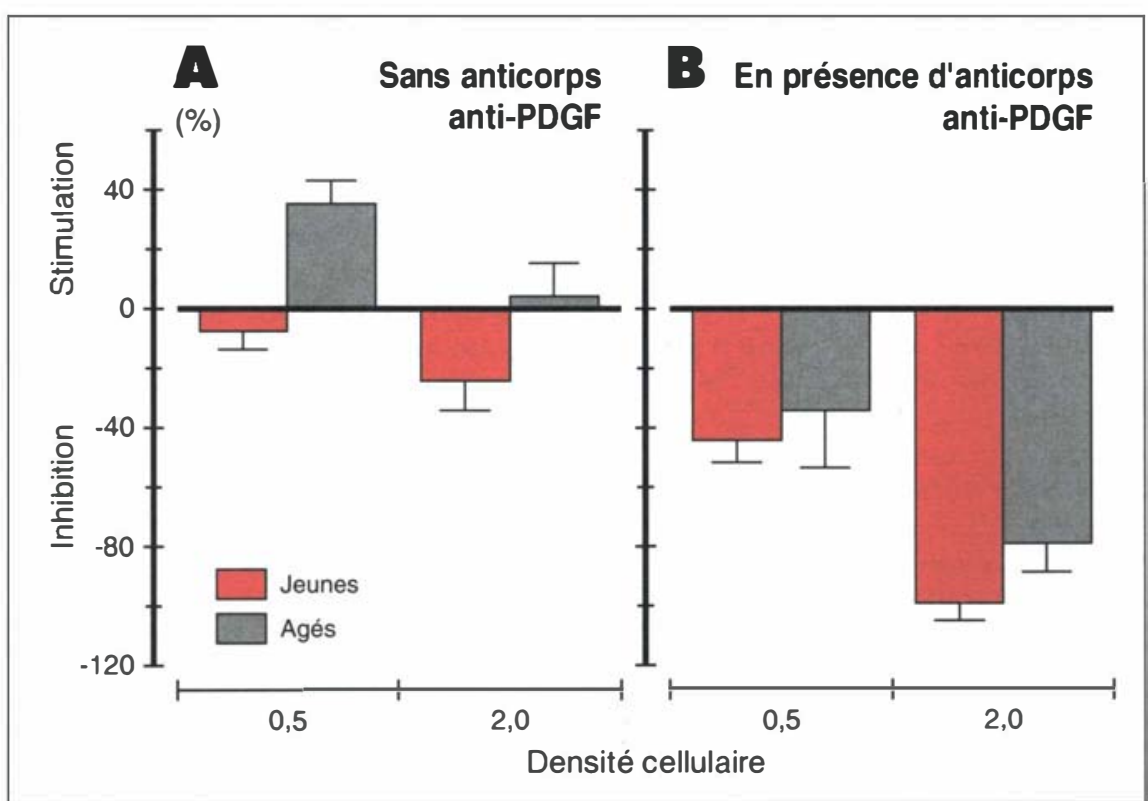

Figure 5. (A) Effets sur la prolifération de cellules cibles, de cellules musculaires lisses (CML) provenant d'aortes de rats jeunes et âgés. A la concentration de $0,5 \times 10^{5}$ cellules $/ \mathrm{ml}$, les lysats de CML âgées expriment un potentiel mitogène, contrairement aux CML jeunes. $A$ une concentration plus forte $\left(2 \times 10^{6}\right.$ cellules $\left./ \mathrm{ml}\right)$, les lysats de CML âgées expriment un effet antiprolifératif moins marqué que les CML jeunes. (B) En présence d'anticorps anti-PDGF, I'effet mitogène des lysats de CML âgées n'est plus observé, et les différences entre l'effet des lysats des CML jeunes et âgées disparaissent.

$m / s n^{\circ} 10$ vol. 9, octobre 93 d'enzymes protéolytiques par les cellules de la paroi), ou des phénomènes de prolifération [29]. Enfin, les cellules mononucléées pourraient participer directement à la dégradation des fibres élastiques en libérant des élastases leucocytaires [30], ou, au contraire, participer à des processus de réparation de la paroi. Toutefois, des études supplémentaires sont nécessaires pour mieux comprendre le rôle de ces cellules dans l'intima d'animaux âgés.

\section{Modifications de la matrice extracellulaire}

Au cours du vieillissement, le contenu en protéines fibreuses de la matrice extracellulaire s'accroît, avec une augmentation du rapport collagène/élastine [6, 31]. La rigidification des artères et la diminution de compliance artérielle des gros troncs artériels qui en résultent jouent un rôle essentiel dans les modifications structurales et fonctionnelles du coeur qui surviennent au cours du vieillissement.

La limitante élastique interne est très épaissie, mais les fibres qui la composent apparaissent fragmentées et moins bien organisées [8, 20]. Des fibrilles et des micro-fibrilles d'élastine apparaissent dans l'intima et participent probablement à la formation de nouvelles lamelles élastiques qui jouxtent la face luminale de limitante élastique interne. Dans la média, on constate au contraire une raréfaction des fibres élastiques qui deviennent irrégulières, fragmentées et dispersées dans l'espace inter-lamellaire (figure 7) [20]. Cette dégénérescence et cette désorganisation de l'élastine dans la média pourraient être la conséquence de l'augmentation, avec l'âge, de la synthèse de certaines protéases, les élastases, présentes dans de nombreuses cellules et notamment dans les cellules musculaires lisses, les fibroblastes et les leucocytes [30]. L'élastase des cellules musculaires lisses de la paroi est de localisation membranaire, et sa synthèse est augmentée par les passages successifs de ces cellules en culture [31]. La dégradation des fibres élastiques, qui est un phénomène très lent dans la paroi de sujets adultes (la demi-vie biolo- 


\section{RÉFÉRENCES}

41. Glagov S, Zarins CK, Giddens DP. Facteurs mécaniques intervenant dans la pathogénie, la localisation et l'évolution des plaques athéroscléreuses. In : Camillieri JP, Berry C, Fiessinger JN, Bariety J, eds. Maladies de la paroi artérielle. Paris : Flammarion, 1987: 187-213.

42. Sarzani R, Brecher P, Chobanian V. Growth factor expression in aorta of normotensive and hypertensive rats. $J$ Clin Invest $1989 ; 83$ : 1404-8.

43. Clowes AW, Reidy MA. Prevention of stenosis after vascular reconstruction : pharmacologic control of intimal hyperplasia. A review. J Vasc Surg 1991; 13:885-91.

44. Nerem RM. Vascular endothelial responses to shear stress. In : Liepsch DW, ed. Blood flow in large arteries : applications to atherogenesis and clinical medecine. Basel : Karger, 1990: 117-24

45. Languille BL, Bendeck MP, Keeley FW. Adaptations of carotid arteries of young and mature rabbits to reduced blood flow. Am J Physiol 1989; 256 : H931-9.

46. Languille BL, O'Donnell F. Reduction in arterial diameter produced by chronic decreases in blood flow are endothelium dependent. Science $1986 ; 231: 405-7$.

47. Haudenschild CC, Chobanian AV. Blood pressure lowering diminishes agerelated changes in the rat aortic intima. Hypertension $1984 ; 6$ : S62-8.

48. Michel JB, Azizi M, Salzmann JL, Lévy BI, Ménard J. Effects of vasodilators on the structure of the aorta in normotensive aging rats. $J$ Hypertension $1987 ; 5$ : S165-8.

49. Stary HC. Evolution and progression of atherosclerotic lesions in coronary arteries of children and young adults. Atherosclerosis $1989 ; 9$ : I19-32.

50. Bullock BC, Clarkson TB, Lehner NDM, et al. Atherosclerosis in Cebus albifrons monkeys. Exp Mol Pathol 1969; 10 : 39-62.

51. Spagnoli LG, Orlandi A, Mauriello A, De Angelis C, Ramaci MT. Age-dependent increase of rabbit aortic atherosclerosis. A morphometric approach. Pathol Res Pract $1992 ; 188$ : 637-42.

52. Fry DL. Mass transport, atherogenesis and risk. Arteriosclerosis 1987; $7: 88-100$.

53. Smith EB, Staples EM. Intimal and medial plasma protein concentrations and endothelial function. Atherosclerosis $1982 ; 41$ :

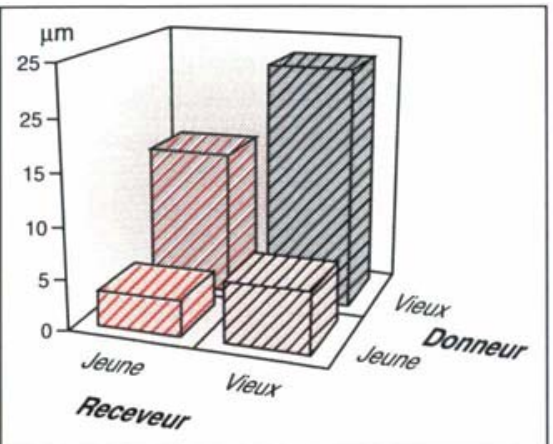

Figure 6. Étude de l'épaississement intimal après désendothélialisation au cours d'expériences de transplantations d'aortes désendothélialisées entre rats jeunes ot âgés. L'épaississement intimal dans l'aorte greffée est représenté en fonction de l'âge du donneur et du receveur. L'importance de l'épaississement intimal après désendothélialisation est liée à l'âge du tissu greffé et non à celui de l'hôte. (D'après Hariri et al. [22].)

gique de ces fibres a pu être évaluée à $40 \mathrm{ans}$ [ [32], serait donc accélérée au cours du vieillissement. La capacité de synthèse de l'élastine dans l'aorte de rats âgés est diminuée [33], si bien que le remplacement des fibres dégradées par des fibres élastiques nouvellement synthétisées pourrait être diminué au cours du vieillissement.

La concentration en collagène augmente dans la média, mais l'organisation des fibres collagènes est moins bien structurée autour des lames élastiques, certaines fibres de collagène étant dispersées dans la matrice [20]. L'intima épaissie des artères âgées est riche en collagène parfois organisé en plaques fibreuses en association avec des protéoglycanes, des fibres élastiques et des cellules musculaires lisses $[7,8]$. Le collagène de la paroi artérielle, comme d'autres protéines de l'organisme, subit au cours du vieillissement des modifications de sa structure chimique $[28,29,34]$. La glycation non enzymatique du collagène entraîne des liaisons chimiques entre les fibres (pontages ou crosslinking), qui peuvent modifier ses propriétés. La glycation du collagène contribue à majorer la rigidité de l'artère et induit une résistance à la protéolyse, notamment par les collagénases, facilitant ainsi l'accumulation du collagène [28]. Ces pontages moléculaires se produisent par réaction chimique entre le collagène et certains sucres, comme le glucose [28, 34], mais aussi les pentoses [35].

D'autres constituants de la matrice extracellulaire semblent modifiés avec l'âge. C'est notamment le cas des protéoglycanes dont la concentration augmente, surtout dans l'intima mais aussi dans la média, avec une modification de la distribution du type de protéoglycane [36]. Les protéoglycanes contenant des glycosaminoglycanes de type héparane-sulfate situés près de la membrane basale de l'endothélium ont une concentration diminuée chez les animaux âgés [36]. Ces composés sont capables d'inhiber la prolifération des cellules musculaires lisses (voir récente revue de Jackson et al. [37]), et leur diminution au cours du vieillissement peut contribuer au développement des cellules musculaires lisses dans l'intima. D'autres types de protéoglycanes à chondroïtine-sulfate ont une concentration dans la matrice qui augmente avec l'âge. Ces composés ont une forte affinité pour les LDL, avec lesquelles ils forment des complexes insolubles [38]. La lipoprotéine a ou $\mathrm{Lp}(\mathrm{a})$, une sous-fraction des LDL qui inhibe l'activité thrombolytique physiologique, est trouvée en quantité importante dans l'espace sousendothélial d'artères âgées [38]. Des pseudo-kystes peuvent être observés dans la matrice extracellulaire de l'artère vieillie. Ces kystes semblent correspondre à des débris cellulaires entourés de membrane cellulaire [7, 8]. Ces lésions, lorsqu'elles sont nombreuses dans la média, sont décrites sous le nom de " média-nécrose disséminée " ou " nécrose laminaire " qui pour certains pourrait contribuer à la genèse de dissections aortiques.

\section{Dynamique du vieillissement artériel}

Il est difficile à l'heure actuelle de décrire avec précision et avec certitude le déroulement et les principales étapes du vieillissement artériel, car les données relatives au vieillissement de la paroi restent assez fragmentaires. Toutefois, à partir des 


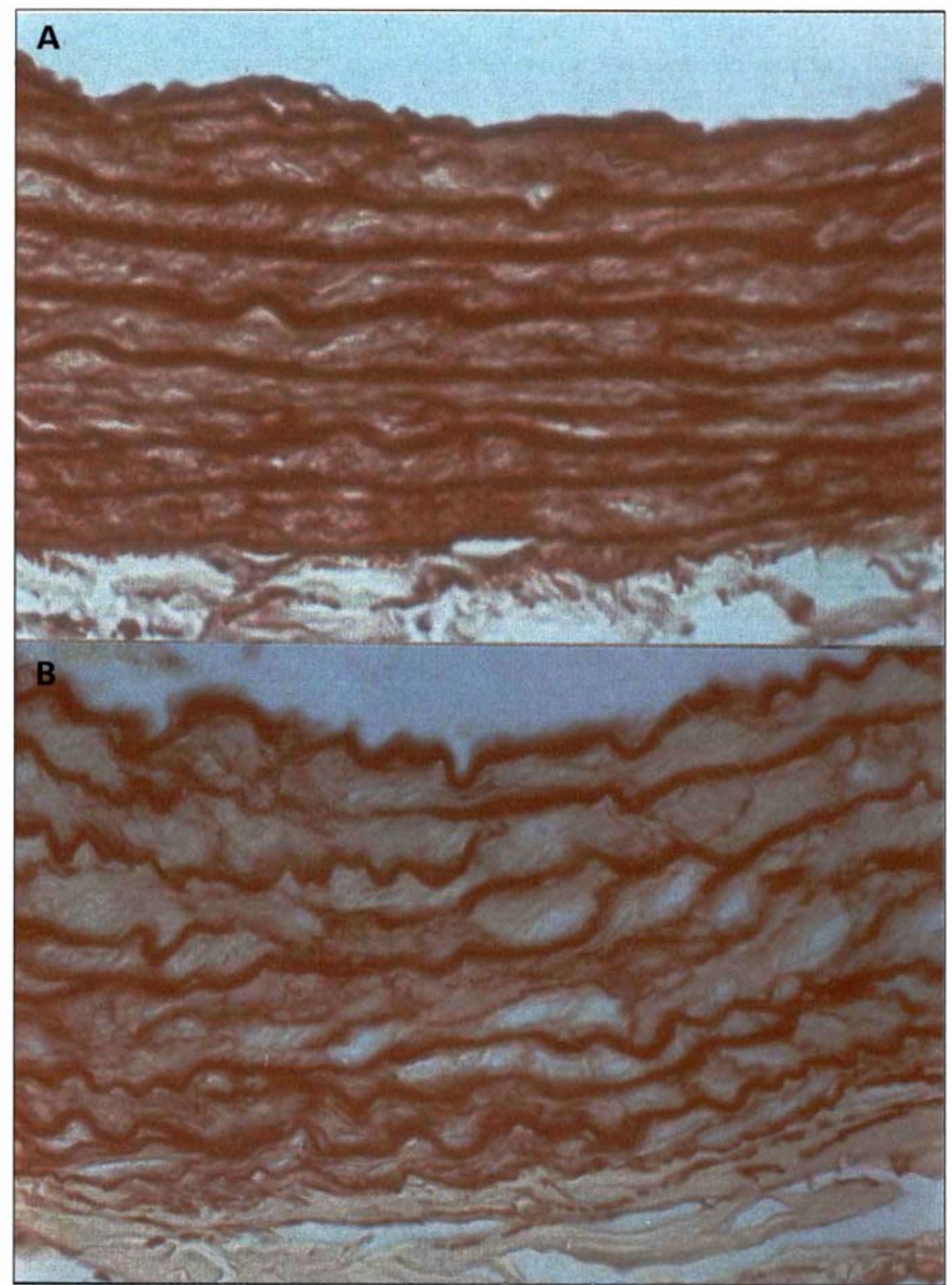

Figure 7. Fibres élastiques de la paroi aortique de rats de 10 mois (A) et de 30 mois (B) sur des coupes transversales de l'aorte thoracique descendante colorées par l'orcéine. Noter la désorganisation des fibres élastiques et la fragmentation de certaines fibres chez le rat âgé (B). L'intima, située entre la lumière et la lame élastique la plus interne (limitante élastique interne), est virtuelle chez le rat jeune (A) et nettement visible et épaissie chez le rat âgé (B). connaissances actuelles, certaines hypothèses ont pu être construites pour tenter de comprendre le déroulement de ce processus. Au cours du vieillissement, se produit de façon très progressive un lent remodelage de la paroi qui peut être compris comme une adaptation de la paroi à de nouvelles conditions mécaniques et/ou biologiques.

\section{La dégénérescence élastique}

La désorganisation et la lyse progressive des fibres élastiques de la média pourraient être le point de départ du remodelage artériel lié à l'âge. Cette élastolyse pourrait être la conséquence d'une usure mécanique provoquée par la contrainte et la déformation que subit l'artère à chaque systole. De telles forces répétitives et cycliques peuvent induire des fractures de fatigue sur de nombreux matériaux, organiques ou non, alors que les mêmes forces, appliquées de façon statique, ne suffisent pas à altérer ces matériaux. Bien que les fibres élastiques soient normalement dégradées et synthétisées en permanence, ce turnover est très lent, et la durée de vie de ces fibres (et donc le temps d'exposition au stress mécanique) est très longue. Aussi, la survenue de fractures de fatigue de ces fibres reste concevable. L'élastolyse de la paroi artérielle pourrait aussi être liée à des phénomènes biologiques, comme la diminution de la synthèse de l'élastine et l'augmentation de sa dégradation enzymatique par les élastases présentes dans les cellules de la paroi, notammment les cellules musculaires lisses, les fibroblastes et les leucocytes [30]. Cette hypothèse est étayée par les études menées sur le vieillissement cutané, qui montrent un certain parallélisme entre l'élastolyse observée dans le derme et celle de la paroi aortique [30]. Toutefois, le rôle des facteurs mécaniques dans l'élastolyse artérielle ne peut être écarté, car l'artère pulmonaire, dont le régime de pression est plus bas, subit au cours du vieillissement une élastolyse bien plus modérée que l'aorte [39].

\section{Remodelage de la paroi}

La perte progressive, avec l'âge, d'une partie du matériel élastique de 


\section{RÉFÉRENCES}

54. Brownlee $\mathrm{M}$, Vlassara $\mathrm{H}$, Cerami $\mathrm{H}$. Nonenzymatic glycosylation products on collagen covalently trap low density lipoprotein. Diabetes 1985 ; 34 : 938-41.

55. Ross R. The pathogenesis of atherosclerosis : an updatc. N Engl J Med 1986 ; 314 : 488-500.

56. Fowkes FGR, Macintyre CCA, Ruckley $\mathrm{CV}$. Increasing incidence of aortic ancuryms in England and Wales. Br Med J $1989 ; 298: 33-5$.

57. Anidjar S, Salzmann JL, Gentric D, Lagneau P, Camilleri JP, Michel JB. Elastase-induced experimental aneurysms in rats. Circulation 1990 ; 82 : 973-81.

58. Solis-Herruzo JA, Brenner DA, Chojkier M. Tumor Necrosis Factor- $\alpha$ inhibits collagen gene transcription and collagen synthesis in cultured human fibroblasts. J Biol Chem 1988; 236 : 5841-5.

59. Kähäri VM, Chen YQ, Su MW, Ramirez F, Uitto J. Tumor Necrosis Factor$\alpha$ and Interferon- $\gamma$ suppress the activation of human type I collagen gene expression by transforming growth factor- $\beta 1$. J Clin Invest $1990 ; 86$ : 1489-95.

60. Liu JM, Davidson JM. The elastogenic effect of recombinant transforming growth factor-beta on porcine aortic smooth muscle cells. Biochem Biophys Res Commun 1988 ; 154 : 895-901.

61. Berk JL, Franzblau C, Goldstein RH. Recombinant interleukin $-1 \beta$ inhibits elastin formation by a neonatal rat lung fibroblast subtype. J Biol Chem 1991; 266 : 3192-7.

62. Shimada Y. Vascular aging and Tumor Necrosis Factor: effects of aging on the interactions between endothelial cells and macrophages. In : Osawa $\mathrm{T}$, Bonavida B, cds. Tumor Necrosis Factor: structure-function relationship and clinical application. Basel : Karger, 1992 : 241-52.

63. Maier JAM, Voulalas P, Roeder D, Maciag T. Extension of the life-span of human endothelial cells by an interleukin-1 $\alpha$ antisense oligomer. Science 1990 ; 249 : 1570-4.

64. Hammes HP, Martin S, Federlin K, Geisen K, Brownlee M. Aminoguanidine treatment inhibits the development of experimental diabctic retinopathy. Proc Natl Acad Sci USA 1991 ; 88 : 11555-8. la média peut expliquer l'augmentation progressive du calibre artériel et un report des contraintes subies par les fibres élastiques sur les fibres de collagène [40]. L'augmentation du calibre artériel est plus marquée dans les gros troncs artériels que dans les artères de moyen calibre qui contiennent moins d'élastine à l'état normal. Cette augmentation du calibre artériel, associée à l'augmentation de la pression artérielle, entraîne une augmentation de la tension pariétale et de la contrainte circonférencielle. Cette dernière valeur est réglée physiologiquement [41], et il est concevable que l'hypertrophie observée au cours du vieillissement tende à normaliser la valeur de la contrainte, comme on l'observe dans l'hypertension artérielle.

La synthèse de collagène aboutissant à une fibrose et à l'épaississement de l'intima est peut-être la conséquence de ces modifications des forces mécaniques exercées sur les fibres, ou sur les cellules endothéliales ou musculaires lisses. En effet, le remodelage de la paroi vasculaire peut être modulé par les forces mécaniques, par l'intermédiaire de la libération de facteurs de croissance ou de facteurs inhibiteurs par les cellules, ou encore par le biais d'interactions matrice-cellules. Par exemple, l'hypertrophie de la paroi artérielle liée à l'hypertension semble être associée à l'expression d'un facteur de croissance, le TGF$\beta$ (transforming growth factor) [42]. L'hypertrophie induite par l'effraction endothéliale semble être dépendante $\mathrm{du}$ basic fibroblast growth factor (bFGF) [43]. Les protéines glycosylées qui s'accumulent dans la paroi artérielle au cours du vieillissement sont capables d'induire la sécrétion de TNF et d'IL1 par les macrophages [29]. Ces facteurs pourraient contribuer au remodelage de la paroi lié au vieillissement, mais on ne dispose actuellement que de peu de données relatives à l'effet de l'âge sur l'expression de ces facteurs de croissance. Par ailleurs, l'endothélium pourrait contribuer directement au contrôle de l'épaississement intimal, en synthétisant certains constituants de la matrice (élastine, glycosaminoglycanes), ou en libérant des facteurs contrôlant la prolifération ou la synthèse cellulaire dans la paroi. Les forces de cisaillement exercées par le flux sanguin sur les cellules endothéliales peuvent moduler les propriétés de ces cellules [44]. L'augmentation du calibre artériel induit une diminution de ces forces de cisaillement, et on peut concevoir, en réaction, une augmentation de l'activité synthétique des cellules endothéliales concourant à l'hypertrophie de l'intima [45]. Il a été rapporté, chez l'animal, que la diminution du calibre artériel s'accompagne d'une atrophie de la média dépendante de l'endothélium [46]. Cette cascade de réactions pourrait être accélérée par l'altération de la fonction de barrière de l'endothélium, conduisant à l'entrée dans l'intima de macromolécules plasmatiques, comme les LDL, et de cellules mononucléées, et par la migration de cellules musculaires lisses dans l'intima sous l'influence de facteurs chimiotactiques. Cette conception du vieillissement de la paroi reste largement hypothétique et devra être confrontée aux résultats des investigations à venir.

\section{Vieillissement et maladies de la paroi artérielle}

\section{Vieillissement et hypertension artérielle}

Les modifications de la paroi liées au vieillissement présentent beaucoup d'analogies avec celles induites par l'hypertension artérielle (Tableau I). Ces analogies laissent penser que l'hypertension pourrait induire un vieillissement accéléré de la paroi artérielle, comme cela a été suggéré par Wolinsky [6] et Glagov [41]. Des contraintes mécaniques intenses pourraient provoquer, en un temps plus bref, des modifications pariétales semblables à celles provoquées par des contraintes moindres, mais appliquées plus longtemps [6]. Toutefois, on peut observer certaines différences entre le remodelage de la paroi lié à l'hypertension et celui lié au vieillissement (Tableau I). L'épaississement intimal et l'altération du tissu élastique sont beaucoup plus marqués dans les artères d'animaux âgés que dans celles d'adultes jeunes hypertendus. En revanche, chez ces derniers, l'hypertrophie de la média et son remaniement fibreux sont plus marqués que chez les animaux âgés nor- 
Tableau I

EFFETS DU VIEILLISSEMENT ET DE L'HYPERTENSION ARTÉRIELLE SUR LA PAROI AORTIQUE

\begin{tabular}{lcc|}
\hline & Vieillissement & HTA \\
\hline Endothélium & & \\
Prolifération & $\uparrow \uparrow$ & $\uparrow \uparrow$ \\
Activité synthétique & $\uparrow \uparrow$ & $\downarrow$ \\
Relaxation dépendante de l'endothélium & $\downarrow \downarrow$ & $\uparrow \uparrow$ \\
Perméabilité aux macromolécules & $\uparrow \uparrow \uparrow$ & \\
Intima & & $\uparrow$ \\
Épaississement intimal & $\uparrow \uparrow \uparrow$ & $\uparrow$ \\
Cellules mononucléées intimales & $\uparrow \uparrow$ & \\
Média & & $\uparrow \uparrow$ \\
Hypertrophie & & $\uparrow \uparrow$ \\
Fibrose & $\uparrow$ & $\uparrow$ \\
Élastolyse & $\uparrow \uparrow \uparrow$ & $\uparrow$ \\
\hline
\end{tabular}

$\uparrow:$ légèrement augmenté $; \uparrow \uparrow:$ augmenté $; \uparrow \uparrow \uparrow:$ très augmenté $; \downarrow \downarrow$ : diminué ; HTA : hypertension artérielle.

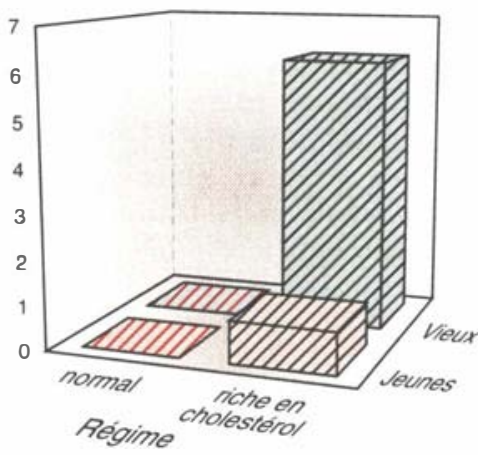

motendus. Ces différences montrent que, au niveau de la paroi artérielle, l'hypertension ne peut pas se résumer entièrement à un vieillissement accéléré. Toutefois, l'étude de l'effet des traitements antihypertenseurs au long cours chez le rat normotendu a montré que le niveau de pression artérielle contribue à moduler le remodelage de la paroi lié à l'âge. En effet, chez les rats normotendus âgés traités plusieurs mois par des antihypertenseurs (et dont la pression artérielle était en moyenne plus basse que celle des rats du même âge non traités), l'épaississement de l'intima n'était plus observé, tout comme la présence de cellules mononucléées dans l'intima [47]. De même, la diminution de la densité en élastine dans la paroi était moins marquée chez les
Figure 8. Volume des lésions athéromateuses évalué par histomorphométrie dans des aortes de lapins jeunes (20 mois) et âgés (4 à 5 ans), soumis ou non à un régime riche en cholestérol. Bien hypercholestérolémiant soit la même (16 mois), les lapins plus âgés développent une athérosclérose plus étendue que les lapins jeunes. (D'après Spagnoli et al. [51].)

rats normotendus ayant vieilli sous traitement antihypertenseur [48].

\section{Vieillissement et athérosclérose}

La prévalence de maladies liées à l'athérosclérose augmente très fortement avec l'âge [3]. La grande frésymptomatiques ou non, chez des personnes très âgées, a laissé penser que l'athérome pourrait être une expression du vieillissement normal. En fait, des lésions d'athérome ont pu être observées à tout âge, notamment chez l'enfant [49]. Inversement, l'absence d'athérome a pu être documentée chez certaines personnes très âgées [2]. A l'heure actuelle, il est largement admis que athérome et vieillissement artériel sont deux processus distincts, même si leur associaque la durée d'exposition au régime quence des lésions athéroscléreuses, tion est fréquente [1]. Une plus longue exposition aux facteurs de risques cardiovasculaires classiques (hypercholestérolémie, hypertension artérielle, tabagisme) explique vraisemblablement une partie des liens entre vieillissement et athérome. Toutefois, les études épidémiologiques montrent que l'âge reste le premier facteur lié au risque cardiovasculaire, et, cela, de façon indépendante des autres facteurs de risque. En fait, des études expérimentales ont montré que, sous régime hypercholestérolémiant, les singes [50] et les lapins [51] âgés développent une athérosclérose plus étendue et plus sévère que des animaux plus jeunes (figure 8). Certaines modifications de la paroi, induites par le vieillissement, sont peut-être aussi à l'origine de ce développement plus facile et/ou plus rapide de l'athérosclérose. L'augmentation de la perméabilité endothéliale aux macromolécules [19] s'accompagnerait d'une plus grande entrée de substances athérogènes dans la paroi et notamment des LDL [52, 53]. L'élastine dégradée se lie aux LDL. Ces complexes étant captés en plus grande quantité par les macrophages, ce phénomène pourrait contribuer à la formation de plaques [30]. Les protéoglycanes de type chondroïtinesulfate, dont la concentration augmente dans l'intima avec l'âge, peuvent également former des complexes avec les LDL [37]. De même, le collagène, qui a subi une glycation non enzymatique, se lie aux LDL et pourrait contribuer aux dépôts de lipides plasmatiques dans les artères âgées [54]. La présence de cellules musculaires lisses ayant un phénotype prolifératif et sécrétoire contribue aux processus d'athérogenèse [55] et la modulation phénotypique de ces cellules avec l'âge pourrait aussi faciliter le développement de lésions d'athérome chez les individus âgés. De plus, il n'est pas exclu que les cellules mononucléées présentes dans l'intima puissent faciliter le développement des plaques en libérant des substances chimiotactiques ou des facteurs de croissance. Enfin, certaines anomalies de la thrombogénicité de l'endothélium liées à l'âge pourraient aussi contribuer à la survenue des maladies liées à l'athérosclérose chez le sujet âgé. 


\section{Vieillissement et anévrismes}

Les anévrismes artériels, caractérisés par une fragmentation du tissu élastique et une dilatation considérable de l'artère, sont observés avec une plus grande fréquence chez les personnes âgées [56]. Chez le rat, le traitement de la paroi aortique par l'élastase s'accompagne du développement d'un anévrisme expérimental [57], ce qui confirme le rôle de l'altération du tissu élastique dans sa physiopathologie. Aussi, on peut concevoir que la dégradation des fibres élastiques liée à l'âge puisse faciliter l'apparition d'un anévrisme si le remodelage de la paroi n'est pas à même de compenser la perte de tissu élastique, notamment en cas d'élastolyse rapide et/ou d'hypertension artérielle sévère.

\section{Perspectives}

Bien que la compréhension des phénomènes à l'origine du vieillissement ne soit pas encore parfaite, les progrès dans le domaine de la biologie de la paroi vasculaire permettent déjà de reconnaître certains processus importants intervenant dans le vieillissement artériel. L'utilisation d'agents pharmacologiques susceptibles de moduler ces processus permettra de mieux saisir leurs rôles respectifs, et, en particulier, de distinguer les altérations dégénératives des modifications adaptatives du vaisseau à son environnement.

Les cytokines, tels le TNF, l'IL1, le TGF- $\beta$ et l'interféron- $\gamma$, sont capables de contrôler l'expression des gènes codant pour la synthèse de collagène $[58,59]$ et de l'élastine $[60,61]$. Elles pourraient, de ce fait, jouer un rôle important dans le remodelage de la paroi lié à l'âge. En effet, le TNF et l'IL1 peuvent être libérés par les macrophages stimulés par des protéines glycosylées produites en abondance au cours du vieillissement [29], et les macrophages provenant d'animaux âgés sont capables de sécréter beaucoup plus de cytokines que ceux d'animaux jeunes [62]. De plus, le blocage intracrine de l'IL1 par des oligonucléotides antisens spécifiques est capable de retarder de façon spectaculaire la perte du potentiel de division des cellules endothéliales vieillissant en culture [63], ce qui ouvre des possibilités de modulation du contrôle génique du vieillissement. L'étude des effets des cytokines sur le vieillissement artériel représente donc une voie de recherche d'avenir dans ce domaine.

Dès à présent, il est possible d'envisager des interventions capables de moduler et de ralentir le vieillissement des artères. La glycation liée à l'âge des protéines de la paroi pourrait être ralentie par des agents pharmacologiques tels que l'aminoguanidine, qui est capable de ralentir la glycation des protéines au cours du diabète expérimental et prévient certaines complications de cette maladie [64]. L'utilisation d'antihypertenseurs chez le rat empêche certaines modifications de la paroi liées à l'âge comme la dégradation élastique [48] et l'épaississement intimal [47]. Un tel traitement est-il capable de faire régresser le vieillissement artériel déjà amorcé ? Peut-il avoir un intérêt chez l'homme, sachant que le vieillissement cardiaque est partiellement conditionné par le vieillissement artériel ? Enfin, des interventions non pharmacologiques, comme l'activité physique soutenue, dont les effets préventifs sur le vieillissement cardiaque sont intéressants chez l'animal, pourraientelles avoir un effet positif sur le vieillissement artériel?

\section{TIRÉS A PART}

\section{J. Belmin.}

\section{Summary}

Aging of the arterial wall

Several changes in structure and function of the arterial wall have been described in association with aging. In the media, elastic fibers show fragmentations, and the collagen/elastin ratio is increased. Glycated proteins that accumulate in aging make collagen fibers more rigid and resistant to proteolysis. Aged smooth muscle cells dedifferentiate into a proliferative and synthetic phenotype, probably responsible for medial and intimal fibrosis. The morphological aspects of the endothelium are not substantially modified in aging, even though some endothelial functions are altered, in particular endothelium-dependent relaxation, prostacyclin release, as well as the endothelial barrier function. The sub-endothelial space is strikingly thickened in aging. This layer contains collagen fibers, proteoglycans, as well as smooth muscle cells, and mononuclear cells normally absent in young arteries. Precise mechanisms and chronology of the aging process in the arterial wall are not well understood, and the main explicative hypotheses are reviewed. Moreover, interactions between aging and arterial diseases - hypertension and atherosclerosis - are discussed. 